\title{
Medial meniscal ramp lesions in ACL-injured elite athletes are strongly associated with medial collateral ligament injuries and medial tibial bone bruising on MRI
}

\author{
Lukas Willinger $^{2} \cdot$ Ganesh Balendra $^{1} \cdot$ Vishal Pai $^{1} \cdot$ Justin Lee $^{1} \cdot$ Adam Mitchell $^{1} \cdot$ Mary Jones $^{1} \cdot$ Andy Williams ${ }^{1}$
}

Received: 22 March 2021 / Accepted: 16 July 2021 / Published online: 3 August 2021

(C) The Author(s) 2021

\begin{abstract}
Purpose Medial menisco-capsular separations (ramp lesions) are typically found in association with anterior cruciate ligament (ACL) deficiency. They are frequently missed preoperatively due to low MRI sensitivity. The purpose of this article was to describe demographic and anatomical risk factors for ramp lesions, and to identify concomitant lesions and define their characteristics to improve diagnosis of ramp lesions on MRI.

Methods Patients who underwent anterior cruciate ligament (ACL) reconstruction between September 2015 and April 2019 were included in this study. The presence/absence of ramp lesions was recorded in preoperative MRIs and at surgery. Patients' characteristics and clinical findings, concomitant injuries on MRI and the posterior tibial slope were evaluated.

Results One hundred patients ( 80 male, 20 female) with a mean age of $22.3 \pm 4.9$ years met the inclusion criteria. The incidence of ramp lesions diagnosed at surgery was $16 \%$. Ramp lesions were strongly associated with injuries to the deep MCL (dMCL, $p<0.01$ ), the superficial medial collateral ligament (sMCL, $p<0.01$ ), and a small medial-lateral tibial slope asymmetry $(p<0.05)$. There was also good correlation between ramp lesions and bone oedema in the posterior medial tibia plateau (MTP, $p<0.05$ ) and medial femoral condyle (MFC, $p<0.05$ ). A dMCL injury, a smaller differential medial-lateral tibial slope than usual, and the identification of a ramp lesion on MRI increases the likelihood of finding a ramp lesion at surgery. MRI sensitivity was $62.5 \%$ and the specificity was $84.5 \%$.

Conclusion The presence on MRI of sMCL and/or dMCL lesions, bone oedema in the posterior MTP and MFC, and a smaller differential medial-lateral tibial slope than usual are highly associated with ramp lesions visible on MRI. Additionally, a dMCL injury, a flatter lateral tibial slope than usual, and the identification of a ramp lesion on MRI increases the likelihood of finding a ramp lesion at surgery. Knowledge of the risk factors and secondary injury signs associated with ramp lesions facilitate the diagnosis of a ramp lesion preoperatively and should raise surgeons' suspicion of this important lesion.

Level of evidence Diagnostic study, Level III.
\end{abstract}

Keywords Ramp lesion · Menisco-capsular separation · Magnetic resonance imaging · Arthroscopy $\cdot$ Risk factors · Association

\section{Introduction}

"Ramp lesions" were first described by Strobel [47] in 1988 to define a menisco-capsular separation of the posterior horn of the medical meniscus (PHMM) form the posteromedial

Andy Williams

williams@fortiusclinic.com

Fortius Clinic, 17 Fitzhardinge St, London W1H 6EQ, UK

2 Department of Trauma Surgery, Technical University of Munich, Ismaninger Straße 22, 81675 Munich, Germany capsule (PMC) and they are most commonly found in association with anterior cruciate ligament (ACL) ruptures $[3$, $6,11,27,31,32,41,45]$. The PHMM is firmly attached to the PMC $[12,52]$ and it acts as a secondary knee stabiliser to resist anterior translation of the medial tibia and thereby external tibial rotation $[1,37,46]$. In the event of an acute ACL injury, the forceful forward displacement of the tibia and subsequent stress on the posteromedial capsule and PHMM can result in posteromedial menisco-capsular injury-a ramp lesion. These occur in $9-34 \%$ of patients with ACL tears at the time of ACL rupture $[3,6,11,27,31$, $32,41,45]$. 
The word 'ramp' refers to the appearance of the synovium/PMC that is seen to sweep proximal and anterior, like a ramp, to the posterior margin of the PHMM when the posteromedial recess is viewed in the flexed knee. In the extended knee, the posterior capsule tightens, and pulled proximally and thereby obliterating the posteromedial recess and making the 'ramp' disappear [46]. It is in the extended position that magnetic resonance imaging (MRI) of the knee is usually undertaken, thus compromising detection of ramp lesions since there is no 'ramp'. This phenomenon may account for the low sensitivity of MRI in identifying ramp lesions, which means ramp lesions are frequently not diagnosed preoperatively $[3,11]$. The intraoperative detection through a systematic arthroscopic exploration, including a direct posteromedial visualization through the intercondylar notch or a direct posteromedial portal, currently remains the gold standard to detect a ramp lesion and can be technically difficult.

Knowledge of the associated factors and secondary injury signs associated with ramp lesions will facilitate the diagnosis of a ramp lesion on MRI. Increased preoperative suspicion of a ramp lesion would be invaluable to the surgeon at time of arthroscopy so that lesions would not be overlooked at surgery. The purpose of this study was to describe demographic and anatomical associated factors for ramp lesions in elite athletes, to identify associated lesions on MRI and define their characteristics. The MRI findings were correlated with operative findings. It was hypothesized that a steeper medial slope is a risk factor for ramp lesions and that bone oedema at the posterior medial tibial plateau (MTP), and medial collateral ligament (MCL) injuries are associated with these injuries, given that it is logical that the injury mechanics causing the ramp lesion are those occurring with AMRI.

\section{Materials and methods}

This study was conducted according to the UK National Health Research Authority guidance and ethically approved by the institution (Fortius Clinic, London, UK) involved. This retrospective cohort study comprised a consecutive series of professional athletes who underwent ACL reconstruction between 2015 and 2019. This group was specifically chosen as they have MRI scans and surgery consistently soon after injury and, therefore, the MRI and surgery would occur with little delay from injury allowing best contemporaneous correlation of clinical, arthroscopic and MRI findings. Patients eligible for inclusion in the study were identified by a review of medical records, and demographic information, injury data, time from injury to MRI and surgery, as well as intraoperative findings were recorded. Patients were excluded if there was any history of previous ipsilateral knee injury or surgery, and any concurrent laxity or surgery of knee ligaments other than the ACL. To allow for high levels of accuracy in evaluation of damage to peripheral structures, only patients with an MRI scan taken within 3 weeks of the ACL injury, and that met the minimum imaging criteria of (1) field strength of 1.5 Tesla or above, (2) three-plane (sagittal, axial and coronal) imaging using water sensitive fat-suppressed sequences (STIR, fat-suppressed proton density or T2-weighted) and (3) slice thickness of $3 \mathrm{~mm}$ or less were included.

\section{Radiological assessment}

Preoperative MRI examinations were acquired from multiple centres. Due to the consequent variation in scanning protocols the sequences used varied. They often included T1-weighted imaging. However, for the purpose of the study, and to maintain consistency, only the fluid sensitive sequences were used for image analysis.

Two radiologists specialized in musculoskeletal imaging with 20 and 25 years of experience, respectively, independently analysed all MRI images. Occurrence of a ramp lesion was recorded if there was fluid signal separating the PMC and the PHMM (Fig. 1). In addition, the presence or absence, and location of bone oedema in the medial compartment was recorded. Bone oedema was defined as increased

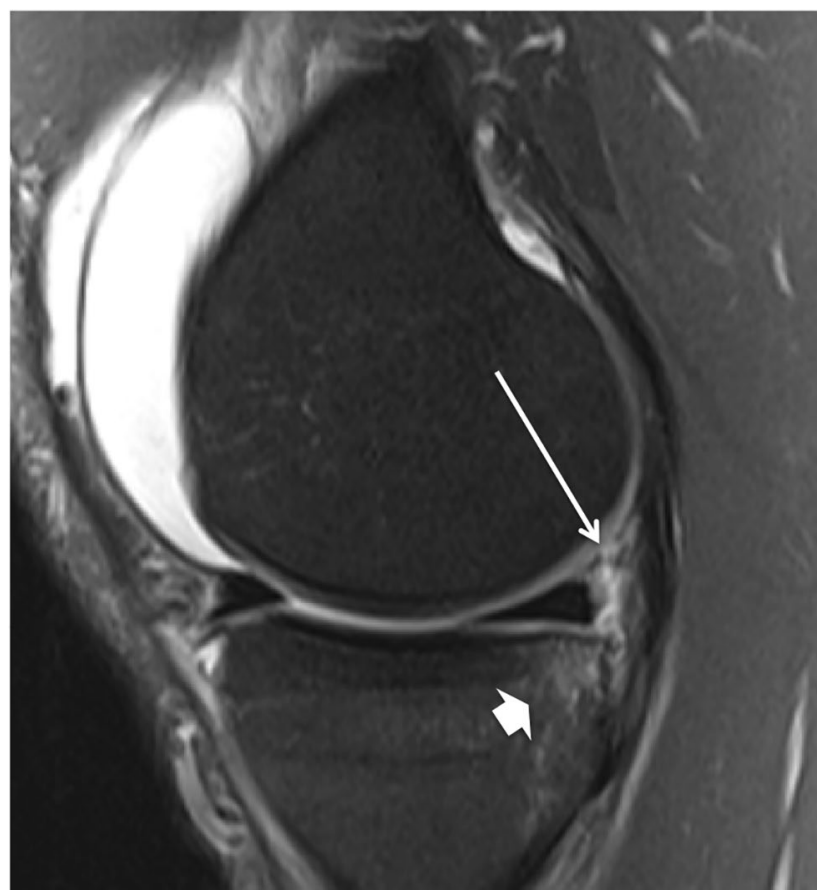

Fig. 1 Sagittal fat-suppressed proton density weighted MRI shows a separation of the posteromedial capsule and the posterior horn of the medial meniscus (ramp lesion, long $\rightarrow$ ) and bone oedema at the posterior medial tibial plateau (thick $\rightarrow$ ) 
signal intensity on the fat-suppressed water sensitive images within the bone. Injuries to the superficial and deep medial collateral ligament (sMCL, and $\mathrm{dMCL}$ ), posterior oblique ligament (POL), lateral collateral ligament (LCL), anterolateral complex including the anterolateral ligament (ALL) and Kaplan fibres (KF), and the menisci were also recorded.

The technique used in this study for measurement of medial and lateral posterior tibial slope has been previously published and validated by other authors [22, 28, 33]. The posterior tibial slope was defined as the difference between the sagittal tibial joint surface orientation and a perpendicular line to the proximal anatomical tibial axis. A larger positive value indicates a steeper posterior tibial slope. Medial-lateral slope differential was calculated by subtracting the medial tibial slope from the lateral medial slope.

\section{Ligament laxity assessment}

All patients were routinely examined under anaesthesia (EUA) at the beginning of surgery by the senior author who is a specialist sports knee surgeon with over 25 years' experience. This included anterior and posterior drawer, Lachman, pivot-shift test, dial test, valgus and varus stress tests. The relevant stress tests were categorized according to International Knee Documentation Committee form (grade I: 3-5 mm, grade II: 5-10 mm, grade III: $>10 \mathrm{~mm}$ ) as laxity differences compared to the healthy contralateral knee [20]. The pivot-shift test was graded as 0 (equal), 1 (glide), 2 (clunk), or 3 (gross).

\section{Arthroscopic assessment}

Standard anteromedial and anterolateral portals were made for ACL reconstruction surgery and a routine diagnostic assessment was made of the suprapatellar pouch, patellofemoral joint, lateral gutter including popliteus tendon and hiatus, medial gutter, medial compartment, intercondylar notch and lateral compartment. The posteromedial compartment and ramp were assessed by advancing a $30^{\circ}$ arthroscope over the anterior surface of the ACL stump into the posteromedial recess through the intercondylar notch. To aid this the knee is held in $30^{\circ}$ flexion with a varus stress applied (Gillquist maneuver $[15,30])$. Some authors recommend inserting the arthroscope via an accessory posteromedial portal to visualise the ramp region [51]. However, this is not the senior author's routine as ramp lesions are revealed as knee flexion was increased (Fig. 2). Occasionally a posteromedial portal is used to insert a probe.

\section{Statistical analysis}

Data were analysed using SPSS statistics software version 23.0 (IBM, New York, USA). Normal distribution was

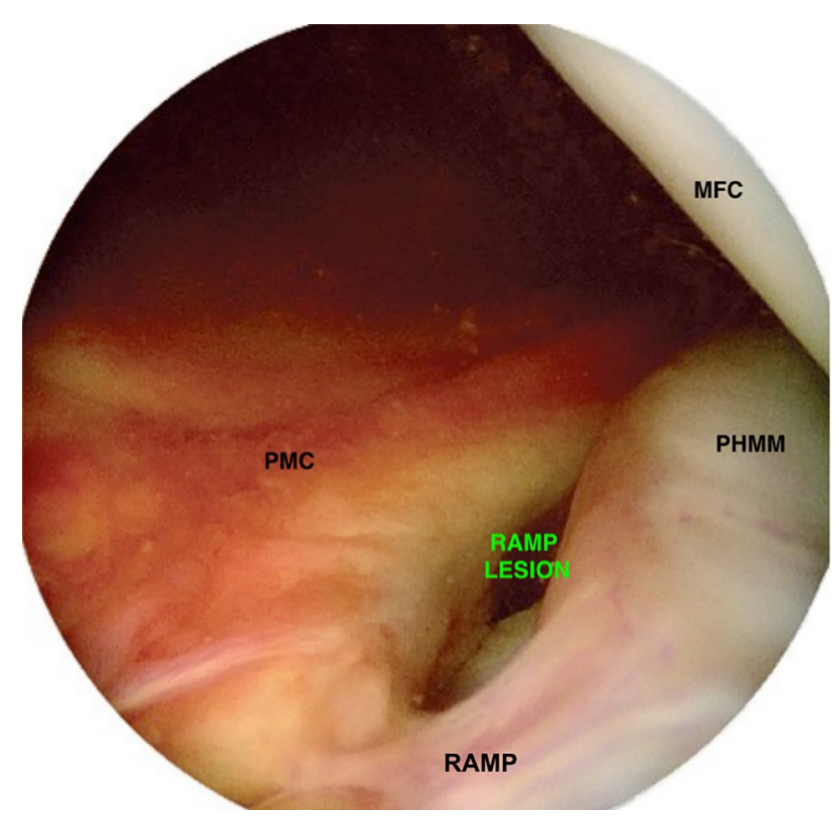

Fig. 2 Arthroscopic trans-notch view through the anterolateral portal with a $30^{\circ}$ camera reveals a menisco-capsular separation (ramp lesion) of a right knee. PHMM posterior horn of the medial meniscus, $P M C$ posterior medial capsule, $M F C$ medial femoral condyle

confirmed by the Shapiro-Wilk test and continuous variables were expressed as mean \pm standard deviation. Chi-squared test or Fisher's exact test was used to analyse for any association between ramp lesions and demographic variables, EUA and other MRI findings. Binomial logistic regression analysis was performed to evaluate the associated factors for the presence of ramp lesions. The included six predictive factors were chosen by background knowledge and were medial and lateral tibial slope, and the presence of sMCL and $\mathrm{dMCL}$ injury, MRI ramp injury and bone oedema at the medial tibial plateau (MPT). Cohen's kappa value has been calculated for inter-rater agreement to detect ramp lesions and inter-rater correlation coefficient (ICC) was calculated for inter-rater reliability for measuring medial and lateral tibial slope A post hoc power analysis revealed an actual power of $82 \%$ for finding differences between two independent proportions ( $\mathrm{p} 1=0.25, \mathrm{p} 2=0.62)$ with a group allocation of 84:16 subjects (intact vs. ramp lesion) (G*Power 3.1). Statistical significance was set at a $p$ value of $<0.05$.

\section{Results}

One hundred and fifty-three patients underwent ACL reconstruction during the study period and of these 100 (80 male and 20 female) with a mean age of $22.3 \pm 4.9$ years met the inclusion criteria. The 53 patients were excluded due to concomitant medial and/or lateral abnormal knee laxity or for failing to meet the minimum imaging criteria. There were 
81 non-contact and 19 contact injuries in 53 right and 47 left knees. All patients were professional athletes and included 60 soccer players, 26 rugby players and 14 players from other sports. The median time between injury and MRI was 2 days (0-21) and 13 days (3-100) between injury and surgery. Cohen's kappa analysis showed excellent agreement between the two readers for the assessment of ramp lesions $(1.00, p<0.001)$. The ICC value for reliability of MRI measurements was 0.892 (95\% CI $0.43-0.925, p<0.01)$ for medial tibial slope and 0.977 (95\% CI 0.966-0.985, $p<0.01$ ) for the lateral tibial slope, indicating excellent agreement.

\section{Incidence of ramp lesions}

The incidence of ramp lesions diagnosed on preoperative MRI was $23 \%$ compared to a 'diagnosis at surgery' rate of $16 \%$. Of these, 15 were repaired and only one was deemed stable and left alone. Hence, the MRI sensitivity to identify a ramp lesion was $62.5 \%$, the specificity was $84.5 \%$, the positive predictive value was $43.5 \%$ and negative predictive value was $92.2 \%$. There was no difference in the timing of the MRI or surgery between the groups with and without ramp lesions.

\section{Association with ramp lesions}

Results of knee laxity EUA and preoperative MRI for the presence of intraoperative ramp lesions are summarized in Tables 1 and 2. Patients' gender, age and injury mechanisms did not correlate with the presence or absence of ramp lesions at surgery.

There was no statistically significant correlation between the presence of ramp lesions and the grade of anterior drawer

Table 1 Patients' EUA results in relation to the presence of intraoperative meniscal ramp lesions

\begin{tabular}{llll}
\hline & \multicolumn{2}{l}{ Meniscus ramp } & \multirow{2}{*}{$p$ value } \\
\cline { 2 - 3 } & Intact & Injured & \\
\hline Anterior drawer test & & \\
$\quad$ Grade I & $69(82.1 \%)$ & $14(87.5 \%)$ & n.s \\
Grade II & $15(17.9 \%)$ & $2(12.5 \%)$ & \\
Lachman test & & & \\
Grade I & $2(2.4 \%)$ & $0(0 \%)$ & n.s \\
Grade II & $31(36.9 \%)$ & $4(25.0 \%)$ & \\
Grade III & $51(60.7 \%)$ & $12(75.0 \%)$ & \\
Pivot shift test* & & & \\
Grade 1 & $25(30.9 \%)$ & $4(26.7 \%)$ & n.s \\
Grade 2 & $47(58.0 \%)$ & $7(46.6 \%)$ & \\
Grade 3 & $9(11.1 \%)$ & $4(26.7 \%)$ & \\
\hline
\end{tabular}

Data is given as numbers (\%)

*Data are only available for 96 patients
Table 2 Concomitant injuries on MRI (i.e. the presence of oedema) in relation to the presence or absence of intraoperative meniscal ramp lesions

\begin{tabular}{|c|c|c|c|}
\hline & \multicolumn{2}{|c|}{ Meniscus ramp } & \multirow[t]{2}{*}{$p$ value } \\
\hline & Intact & Injured & \\
\hline \multicolumn{4}{|l|}{ Superficial MCL } \\
\hline Intact & $39(46.4 \%)$ & $1(6.3 \%)$ & \multirow[t]{2}{*}{$p=0.002$} \\
\hline Injured & $45(53.6 \%)$ & $15(93.8 \%)$ & \\
\hline \multicolumn{4}{|l|}{ Deep MCL } \\
\hline Intact & $63(75.0 \%)$ & $6(37.5 \%)$ & \multirow[t]{2}{*}{$p=0.006$} \\
\hline Injured & $21(25.0 \%)$ & $10(62.5 \%)$ & \\
\hline \multicolumn{4}{|l|}{ POL } \\
\hline Intact & $75(89.3 \%)$ & $14(87.5 \%)$ & \multirow[t]{2}{*}{ n.s } \\
\hline Injured & $9(10.7 \%)$ & $2(12.5 \%)$ & \\
\hline \multicolumn{4}{|l|}{ LCL } \\
\hline Intact & $78(92.9 \%)$ & $15(93.8 \%)$ & \multirow[t]{2}{*}{ n.s } \\
\hline Injured & $6(7.1 \%)$ & $1(6.3 \%)$ & \\
\hline \multicolumn{4}{|l|}{ Kaplan fibres } \\
\hline Intact & $36(42.9 \%)$ & $3(18.8 \%)$ & \multirow[t]{2}{*}{ n.s } \\
\hline Injured & $48(57.1 \%)$ & $13(81.3 \%)$ & \\
\hline \multicolumn{4}{|l|}{ Anterolateral ligament } \\
\hline Intact & $67(79.8 \%)$ & $12(75.0 \%)$ & \multirow[t]{2}{*}{ n.s } \\
\hline Injured & $17(20.2 \%)$ & $4(25.0 \%)$ & \\
\hline \multicolumn{4}{|l|}{ Medial meniscus } \\
\hline Intact & $56(66.7 \%)$ & $10(62.5 \%)$ & \multirow[t]{2}{*}{ n.s } \\
\hline Injured & $28(33.3 \%)$ & $6(37.5 \%)$ & \\
\hline \multicolumn{4}{|l|}{ Lateral meniscus } \\
\hline Intact & $37(44.0 \%)$ & $8(50.0 \%)$ & \multirow[t]{2}{*}{ n.s } \\
\hline Injured & $47(56.0 \%)$ & $8(50.0 \%)$ & \\
\hline \multicolumn{4}{|l|}{ MTP bone oedema } \\
\hline Absent & $40(47.6 \%)$ & $2(12.5 \%)$ & \multirow[t]{2}{*}{$p=0.012$} \\
\hline Present & $44(52.4 \%)$ & $14(87.5 \%)$ & \\
\hline \multicolumn{4}{|l|}{ MFC bone oedema } \\
\hline Absent & $60(71.4 \%)$ & $7(43.8 \%)$ & \multirow[t]{2}{*}{$p=0.043$} \\
\hline Present & $24(28.6 \%)$ & $9(56.3 \%)$ & \\
\hline Medial tibial slope $\left({ }^{\circ}\right)$ & $3.3 \pm 2.3$ & $3.7 \pm 2.3$ & n.s \\
\hline Lateral tibial slope $\left({ }^{\circ}\right)$ & $7.0 \pm 3.7$ & $5.4 \pm 2.9$ & n.s \\
\hline Tibial slope asymmetry $\left(^{\circ}\right)$ & $3.8 \pm 3.9$ & $1.7 \pm 2.8$ & $p=0.044$ \\
\hline
\end{tabular}

Data is given as numbers (\%)

$M C L$ medial collateral ligament, $P O L$ posterior oblique ligament, $L C L$ lateral collateral ligament, MTP medial tibial plateau, $M F C$ medial femoral condyle

test, Lachman test and pivot-shift test (Table 1). However, 12 of 16 patients $(75 \%)$ with a ramp lesion had a grade III Lachman test.

93.7\% of patients with ramp lesions exhibited concomitant sMCL injury (the presence of oedema in the sMCLbut with normal clinical laxity) on MRI. Ramp lesions are strongly associated with injuries to the sMCL ( $p=0.002$, OR $13.000,95 \%$ CI $1.642-102.936)$, and the dMCL ( $p=0.006$, OR 5.000, 95\% CI 1.621-15.419). There was also a strong 
correlation between ramp lesions and bone oedema in the posterior MTP ( $p=0.012$, OR 6.364, 95\% CI 1.361-29.750) and MFC ( $p=0.043$, OR 3.214, 95\% CI 1.075-9.611). In addition, differential medial-lateral posterior tibial slope was significantly smaller in patients with a ramp lesion compared to patients with an intact meniscus ramp $(p<0.05)$. Ramp lesions were not associated with oedema in the POL, LCL, Kaplan fibres, or ALL, nor medial or lateral meniscus lesions (Table 2).

\section{Associated factors for ramp lesions}

Associated factors associated with the ramp lesions seen at arthroscopy were identified with logistic regression analysis with backward elimination (Table 3 ). The presence of a MRI ramp lesion, dMCL injury and a flatter lateral tibial slope was statistically significant associated factors for the presence of ramp lesion at surgery. Finding a ramp lesion intraoperatively is more likely if it is detected on MRI (OR 14.87). The risk of having a ramp lesion was significantly raised by a simultaneous dMCL (OR 6.45). A flatter lateral tibial slope increased the risk of having a ramp lesion with each degree by 1.3 times.

\section{Discussion}

The main findings of this study were that the presence on MRI of posterior MTP and MFC oedema, SMCL and dMCL lesions and a smaller tibial slope asymmetry $\left(1.7^{\circ}\right.$ vs. $\left.3.8^{\circ}\right)$ are highly associated with ramp lesions. Additionally, with binomial logistic regression analysis, a dMCL injury, a flatter lateral tibial slope and the identification of a ramp lesion on MRI significantly increased the likelihood of finding a ramp lesion at surgery.

The medial meniscus, with its firm attachment to the tibia via the menisco-tibial ligament, and specifically the posterior horn, is a secondary restraint to anterior tibial translation and external rotation of the knee. Its function becomes even more important in ACL-deficient knees [1, 2, 26, 35]. Biomechanical studies have demonstrated that ramp lesions in addition to ACL deficiency, increases anteroposterior instability and external rotational instability $[1,46]$. AMRI has also been found in clinical studies [6, 32, 49]. There is growing scientific evidence and consensus among knee surgeons that ramp lesions should be sought, identified and repaired [7, 38, 40, 50]. Repair of ramp lesions is safe [21] and restores normal knee kinematics when combined with ACL reconstruction in in-vitro studies [46]. Therefore, it is vital to detect ramp lesions, both on MRI and at surgery, and repair them at the time of ACL reconstruction or risk ongoing pain, instability and ACL graft failure due to overload. Traditionally ramp lesions were not detected as they cannot easily be identified when viewing the posterior medial meniscus with the arthroscope placed anteriorly in the medial compartment. The diagnostic requires to view the ramp region with the arthroscope in the posteromedial recess using the intercondylar approach or an additional posteromedial portal. As recommended, this inspection must be done routinely during ACL reconstruction procedures. MRI identification of a ramp lesion and its associated factors will alert a surgeon to focus to the posteromedial area.

As an identified ramp lesion on MRI increased the chance of finding it at surgery by 13.6 times it emphasises the importance of this knowledge. This study found that MRI had a moderate sensitivity (62.5\%) but high specificity $(84.5 \%)$, and a high negative predictive value $(92.2 \%)$ in detecting ramp lesions. The published rates for MRI sensitivity in identifying ramp lesions are 48-90\% [3, 11, 19, 27, 29, $49,56]$ with 3 T MRI possibly superior to 1.5 T MRI scans [19]. Arner et al. [3] compared results of three MRI readers with arthroscopic findings. Their MRI sensitivity varied between 53.9 and $84.6 \%$. This is similar to the findings of the present study, which showed a much higher inter-rater agreement, highlighting the benefit of having experienced MSK radiologists and of applying specific MRI criteria for ramp lesions. MRI specificity was reported to be over $90 \%$ in several studies $[3,19,27]$, which is similar to the findings in this study of $84.5 \%$. Overall these results indicate that preoperative MRI is not accurate enough to detect all ramp lesions but is an excellent modality to exclude the presence of ramp lesions. Therefore, routine inspection of the ramp region in the posteromedial recess during arthroscopy in knees with ACL injury is advocated.
Table 3 Logistic regression analysis shows the association between the presence of an intraoperative ramp lesion and clinical and radiological factors

\begin{tabular}{lllrl}
\hline Factor & Odds ratio & 95\% CI & \multicolumn{1}{c}{$B \pm$ SE } & $p$ value \\
\hline Lateral tibial slope & 0.761 & $0.606-0.955$ & $-0.274 \pm 0.116$ & $\boldsymbol{p}=\mathbf{0 . 0 1 9}$ \\
dMCL injury & 6.449 & $1.459-28.504$ & $1.864 \pm 0.758$ & $\boldsymbol{p}=\mathbf{0 . 0 1 4}$ \\
MRI ramp lesion & 14.870 & $2.914-75.872$ & $2.699 \pm 0.321$ & $\boldsymbol{p}=\mathbf{0 . 0 0 1}$ \\
Constant value & & & $-4.041 \pm 1.339$ & $\boldsymbol{p}=\mathbf{0 . 0 1 8}$ \\
\hline
\end{tabular}

Odds ratio describes the risk of exhibiting a ramp lesion. Nagelkerke $R^{2}=0.48$

$C I$ confidence interval, $d M C L$ deep medial collateral ligament 
The incidence of ramp lesions in ACL-injured knees at surgery in a professional athlete's population in this study is $16 \%$. This is consistent with the published literature in which the incidence is reported to range from 9 to $34 \%$ [3, $6,11,19,27,29,31,41,56]$. The difference of ramp lesions' prevalence between contact and non-contact injuries was not significant in the present study, but has been described previously [41]. It is important to mention that although good views were obtained at surgery, it is possible that simply viewing the 'ramp' region with a $30^{\circ}$ arthroscope passed into the posteromedial recess via the intercondylar notch and not using a posteromedial portal that the some ramp lesions could have been missed and, therefore, the incidence is under-reported.

In the present study, the presence of ramp lesions is strongly associated with concurrent MRI injury to the SMCL and especially the dMCL. Interestingly, ramp lesions were not associated with damaged to the posteromedial capsule, what could have been expected due to the close anatomical relation [8]. This finding links injuries to the posterior menisco-capsular junction of the medial meniscus with injuries to the medial collateral ligaments [23]. One of the roles of the MCL is resistance to anterior translation of the medial tibial plateau/external rotation for which the dMCL is the primary restraint between $0^{\circ}$ and $60^{\circ}$ knee flexion $[4$, $39,53,54]$ as well as valgus, for which the sMCL is the primary restraint. The association of concomitant injury to the $\mathrm{dMCL}$ and $\mathrm{SMCL}$ and ramp lesions is suggestive of a specific injury mechanism causing the ramp lesion as well as injury to the dMCL and sMCL. To injure these structures together logically there must be significant anterior translation and/or external rotatory subluxation of the medial tibial plateau during the event of an ACL rupture, since these loads are resisted by the posterior medial meniscus, dMCL and SMCL. This might cause the medial femoral condyle to move posteriorly and ride over the medial meniscus thereby stretching the PMC in the posteromedial recess to the point of failure [16]. In fact, 15 of 16 patients (93.7\%) with ramp lesions had a sprain of the sMCL and $62.5 \%$ a lesion of the dMCL, which is firmly bound to the mid-portion of the medial meniscus. In contrast, the POL was intact in $87.5 \%$ patients, further reinforcing the idea that this injury is due to an anterior translation of the medial tibia /external rotation injury mechanism as the POL resists internal rotation in the knee close to full extension. Furthermore, bone oedema at the posterior MTP and the MFC was also correlated with ramp lesions, and $87.5 \%$ of cases with proven ramp lesions had MTP bone oedema, which is in keeping with the same mechanism. This finding is in agreement with previous studies that reported posteromedial tibial bone oedema as an important secondary MRI finding in conjunction with ramp lesions [5, 11, 27, 29]. DePhillipo et al. [11] found MTP bone bruises in $72 \%$ of their patients diagnosed with ramp lesions. In contrast, Hatayama et al. only found bone contusions in $38 \%$ of their patients with ramp lesions and the incidence of bone contusion did not differ from patients with an intact medial meniscus [19]. The higher incidence in the present study may reflect the patient population: perhaps injuries in professional athletes are more severe.

The present study also showed that a smaller posterior tibial slope asymmetry was associated with the ramp lesion and, from the logistic regression analysis, that a steeper posterior lateral tibial slope decreased the risk for them. In contrast, increased lateral tibial slope is associated with ACL rupture [10, 14, 18]. The authors of this present study believe that a higher lateral tibial slope predisposes to the lateral femoral condyle subluxing off the posterior lateral tibia causing ACL rupture and the classic lateral compartment distal femoral and posterior tibial bone bruises. In such cases the abnormal motion is predominantly in the lateral compartment with the centre of axial rotation on the medial tibial plateau leaving the ramp intact as the MFC does not move posteriorly. Conversely in knees with less lateral posterior slope laterally, and thus less differential medial/lateral posterior slope, with anterior tibial displacement the femur will have less tendency to move posteriorly just in the lateral component and will also do so medially hence loading the PHMM and PMC. Our results are similar to the findings of Kim et al. [27] who associated ramp lesions with a steeper medial and flatter lateral slope. Also Song et al. found a higher incidence of ramp lesions in patients with an increased medial slope [44].

With regard to clinical examination, Bollen described a correlation of ramp lesions with anteromedial rotatory subluxation [6]. The author noticed an increased anterior movement of the medial tibial plateau when the foot was externally rotated in $90^{\circ}$ knee flexion [6] (i.e. Slocum test [42]). Ramp lesions have also been associated with a higher side-to-side difference of anterior translation examined with a KT-2000 [49] and a higher incidence of grade III pivotshift [32]. This study could not identify a statistically significant association between the presence of ramp lesions and a higher grade of Lachman test, anterior drawer test or pivot-shift test. However, 12 of the 16 knees with a ramp lesion had grade 3 Lachman tests and the other 4 were grade 2. Failure to reach statistical significance could, therefore, be a type 2 error as $20 \%$ of studies suffer from failure to show a difference statistically when one actually exists [13]. Unfortunately, the Slocum test for anteromedial rotatory instability was not routinely performed during the present study.

This study has several limitations. All patients were professional athletes and, therefore, do not represent a typical patient cohort compared to most clinical practices, and their injury patterns and rates of ramp lesion may not be the same as in other patient groups. These patients were, however, chosen to ensure the best quality of MRI imaging, and scans 
sufficiently soon after injury to document the full extent of injury to intra-articular structures and the soft tissue envelope as they have more immediate access to MRI examination. Since these patients tend to have early surgery direct correlation of MRI findings with arthroscopic diagnosis is possible. The situation can change as delay might allow time for healing of ramp lesions. This does bring into to question the possibility that early surgery increases diagnosis and possible over treatment of ramp lesions that might otherwise heal. Furthermore, the size and category of ramp lesions (stable or unstable) was not further classified and analysed.

In addition, MRIs were, however, acquired in various institutions with different protocols that could affect scan quality, but minimum imaging requirements were applied for inclusion in the study design to allow for reliable analysis, which was reflected by high inter-rater agreement. An obvious issue is that whilst abnormal (high) signal represents injury to tissue, the integrity or otherwise of that tissue cannot be certain. Furthermore, spread of oedema / hematoma might, by MRI criteria, imply injury to soft tissues that are, in fact, intact. Again, this risk is mitigated by the scans mainly being undertaken 2 or 3 days from injury.

The results from the present study emphasize the relation between ramp lesions and damage to the medial soft tissue envelope, hence, indicating a likely external rotation injury mechanism in some ACL ruptures. This should raise awareness of possible AMRI in these patients which need to be carefully assessed through clinical examination and addressed in the operating room.

\section{Conclusion}

In cases of acute ACL rupture, on MRI the presence of bone oedema in the MFC and posterior MTP, sMCL and dMCL lesions, and a smaller differential medial-lateral tibial slope are highly associated with ramp lesions. Additionally, a dMCL injury, a flatter lateral tibial slope, and the identification of a ramp lesion on MRI increase the likelihood of finding a ramp lesion at surgery according to logistic regression analysis. Preoperative MRI has only moderate sensitivity, but high specificity, and a high negative predictive value for detection of ramp lesions.

Author contributions LW, GB and AW designed the study, performed the statistical analysis and wrote the manuscript. LW drafted the manuscript. AW was responsible for clinical examination and the surgical treatment. VP, AM and JL collected the data and performed the radiological and MRI evaluation. MJ helped to design the study, assisted with data collection, statistical analysis and data interpretation and critically reviewed the manuscript. All authors read and approved the final manuscript.
Funding Open Access funding enabled and organized by Projekt DEAL. No funding was received for this study.

\section{Declarations}

Conflict of interests The authors did not receive financial support or other benefits from commercial sources for the work reported in this manuscript or any other financial support that could create a potential or the appearance of a conflict of interest regarding to the work.

Ethics approval Approval to undertake the study was given by the institution involved in line with the UK Health Research Authority guidance.

Open Access This article is licensed under a Creative Commons Attribution 4.0 International License, which permits use, sharing, adaptation, distribution and reproduction in any medium or format, as long as you give appropriate credit to the original author(s) and the source, provide a link to the Creative Commons licence, and indicate if changes were made. The images or other third party material in this article are included in the article's Creative Commons licence, unless indicated otherwise in a credit line to the material. If material is not included in the article's Creative Commons licence and your intended use is not permitted by statutory regulation or exceeds the permitted use, you will need to obtain permission directly from the copyright holder. To view a copy of this licence, visit http://creativecommons.org/licenses/by/4.0/.

\section{References}

1. Ahn JH, Bae TS, Kang KS, Kang SY, Lee SH (2011) Longitudinal tear of the medial meniscus posterior horn in the anterior cruciate ligament-deficient knee significantly influences anterior stability. Am J Sports Med 39:2187-2193

2. Allen CR, Wong EK, Livesay GA, Sakane M, Fu FH et al (2000) Importance of the medial meniscus in the anterior cruciate ligament-deficient knee. J Orthop Res 18:109-115

3. Arner JW, Herbst E, Burnham JM, Soni A, Naendrup JH et al (2017) MRI can accurately detect meniscal ramp lesions of the knee. Knee Surg Sports Traumatol Arthrosc 25:3955-3960

4. Ball S, Stephen JM, El-Daou H, Williams A, Amis AA (2020) The medial ligaments and the ACL restrain anteromedial laxity of the knee. Knee Surg Sports Traumatol Arthrosc 28:3700-3708

5. Beel W, Mouton C, Tradati D, Nuhrenborger C, Seil R (2021) Ramp lesions are six times more likely to be observed in the presence of a posterior medial tibial bone bruise in ACL-injured patients. Knee Surg Sports Traumatol Arthrosc. https://doi.org/ 10.1007/s00167-021-06520-z

6. Bollen SR (2010) Posteromedial meniscocapsular injury associated with rupture of the anterior cruciate ligament: a previously unrecognised association. J Bone Jt Surg Br 92:222-223

7. Bumberger A, Koller U, Hofbauer M, Tiefenboeck TM, Hajdu S et al (2020) Ramp lesions are frequently missed in ACL-deficient knees and should be repaired in case of instability. Knee Surg Sports Traumatol Arthrosc 28:840-854

8. Cavaignac E, Sylvie R, Teulieres M, Fernandez A, Frosch KH et al (2021) What is the relationship between the distal semimembranosus tendon and the medial meniscus? A gross and microscopic analysis from the SANTI study group. Am J Sports Med 49:459-466

9. Church S, Keating JF (2005) Reconstruction of the anterior cruciate ligament: timing of surgery and the incidence of meniscal tears and degenerative change. J Bone Jt Surg Br 87:1639-1642 
10. Dare DM, Fabricant PD, McCarthy MM, Rebolledo BJ, Green DW et al (2015) Increased lateral tibial slope is a risk factor for pediatric anterior cruciate ligament injury: an MRI-based casecontrol study of 152 patients. Am J Sports Med 43:1632-1639

11. DePhillipo NN, Cinque ME, Chahla J, Geeslin AG, Engebretsen $L$ et al (2017) Incidence and detection of meniscal ramp lesions on magnetic resonance imaging in patients with anterior cruciate ligament reconstruction. Am J Sports Med 45:2233-2237

12. DePhillipo NN, Moatshe G, Chahla J, Aman ZS, Storaci HW et al (2018) Quantitative and qualitative assessment of the posterior medial meniscus anatomy: defining meniscal ramp lesions. Am J Sports Med. https://doi.org/10.1177/03635465188142583635 46518814258

13. Domb BG, Sabetian PW (2021) When no difference does not mean there is no difference: the blight of the type II error. Arthroscopy. https://doi.org/10.1016/j.arthro.2021.01.057

14. Feucht MJ, Mauro CS, Brucker PU, Imhoff AB, Hinterwimmer S (2013) The role of the tibial slope in sustaining and treating anterior cruciate ligament injuries. Knee Surg Sports Traumatol Arthrosc 21:134-145

15. Gillquist J, Hagberg G, Oretorp N (1979) Arthroscopic examination of the posteromedial compartment of the knee joint. Int Orthop 3:13-18

16. Grassi A, Tosarelli F, Agostinone P, Macchiarola L, Zaffagnini $S$ et al (2020) Rapid posterior tibial reduction after noncontact anterior cruciate ligament rupture: mechanism description from a video analysis. Sports Health 12:462-469

17. Hamberg P, Gillquist J, Lysholm J (1983) Suture of new and old peripheral meniscus tears. J Bone Jt Surg Am 65:193-197

18. Hashemi J, Chandrashekar N, Mansouri H, Gill B, Slauterbeck JR et al (2010) Shallow medial tibial plateau and steep medial and lateral tibial slopes: new risk factors for anterior cruciate ligament injuries. Am J Sports Med 38:54-62

19. Hatayama K, Terauchi M, Saito K, Aoki J, Nonaka S et al (2018) Magnetic resonance imaging diagnosis of medial meniscal ramp lesions in patients with anterior cruciate ligament injuries. Arthroscopy 34:1631-1637

20. Hefti F, Muller W, Jakob RP, Staubli HU (1993) Evaluation of knee ligament injuries with the IKDC form. Knee Surg Sports Traumatol Arthrosc 1:226-234

21. Heilpern G, Stephen J, Ball S, Amis AA, Williams A (2018) It is safe and effective to use all inside meniscal repair devices for posteromedial meniscal 'ramp' lesions. Knee Surg Sports Traumatol Arthrosc 26:2310-2316

22. Hudek R, Schmutz S, Regenfelder F, Fuchs B, Koch PP (2009) Novel measurement technique of the tibial slope on conventional MRI. Clin Orthop Relat Res 467:2066-2072

23. Hughston JC, Andrews JR, Cross MJ, Moschi A (1976) Classification of knee ligament instabilities. Part I. The medial compartment and cruciate ligaments. J Bone Jt Surg Am 58:159-172

24. Indelicato PA, Bittar ES (1985) A perspective of lesions associated with ACL insufficiency of the knee: a review of 100 cases. Clin Orthop Relat Res 198:77-80

25. Keene GC, Bickerstaff D, Rae PJ, Paterson RS (1993) The natural history of meniscal tears in anterior cruciate ligament insufficiency. Am J Sports Med 21:672-679

26. Kent RN, Imhauser CW, Thein R, Marom N, Wickiewicz TL et al (2020) Engagement of the secondary ligamentous and meniscal restraints relative to the anterior cruciate ligament predicts anterior knee laxity. Am J Sports Med 48:109-116

27. Kim SH, Seo HJ, Seo DW, Kim KI, Lee SH (2020) Analysis of risk factors for ramp lesions associated with anterior cruciate ligament injury. Am J Sports Med 48:1673-1681

28. Kolbe R, Schmidt-Hebbel A, Forkel P, Pogorzelski J, Imhoff AB et al (2018) Steep lateral tibial slope and lateral-to-medial slope asymmetry are risk factors for concomitant posterolateral meniscus root tears in anterior cruciate ligament injuries. Knee Surg Sports Traumatol Arthrosc 27:2585-2591

29. Kumar NS, Spencer T, Cote M, Arciero RA, Edgar C (2018) Is edema at the posterior medial tibial plateau indicative of a ramp lesion? An examination of 307 patients with anterior cruciate ligament reconstruction and medial meniscal tears. Orthop J Sports Med 6:2325967118780089

30. Lee JY, Chia ZY, Jiang L, Ang B, Chang P (2020) A review of the Gillquist Maneuver: modifications for a safer and easily reproducible approach for knee transintercondylar notch posterior compartment arthroscopy. Arthrosc Tech 9:e435-e438

31. Liu X, Feng H, Zhang H, Hong L, Wang XS et al (2011) Arthroscopic prevalence of ramp lesion in 868 patients with anterior cruciate ligament injury. Am J Sports Med 39:832-837

32. Mouton C, Magosch A, Pape D, Hoffmann A, Nuhrenborger C et al (2020) Ramp lesions of the medial meniscus are associated with a higher grade of dynamic rotatory laxity in ACL-injured patients in comparison to patients with an isolated injury. Knee Surg Sports Traumatol Arthrosc 28:1023-1028

33. Naendrup JH, Drouven SF, Shaikh HS, Jaecker V, Offerhaus C et al (2020) High variability of tibial slope measurement methods in daily clinical practice: comparisons between measurements on lateral radiograph, magnetic resonance imaging, and computed tomography. Knee 27:923-929

34. Noyes FR, Chen RC, Barber-Westin SD, Potter HG (2011) Greater than 10-year results of red-white longitudinal meniscal repairs in patients 20 years of age or younger. Am J Sports Med 39:1008-1017

35. Papageorgiou CD, Gill JE, Kanamori A, Fenwick JA, Woo SL et al (2001) The biomechanical interdependence between the anterior cruciate ligament replacement graft and the medial meniscus. Am J Sports Med 29:226-231

36. Papasterigou SG, Koukoulias NE, Mikalef P, Ziogas E, Voulgaropoulos H (2007) Meniscal tears in the ACL-deficient knee: correlation between meniscal tears and the timing of ACL reconstruction. Knee Surg Sports Traumatol Arthrosc 15:1438-1444

37. Peltier A, Lording T, Maubisson L, Ballis R, Neyret $P$ et al (2015) The role of the meniscotibial ligament in posteromedial rotational knee stability. Knee Surg Sports Traumatol Arthrosc 23:2967-2973

38. Reider B (2017) Ramped up. Am J Sports Med 45:1001-1003

39. Robinson JR, Bull AM, Thomas RR, Amis AA (2006) The role of the medial collateral ligament and posteromedial capsule in controlling knee laxity. Am J Sports Med 34:1815-1823

40. Seil R (2018) Medial meniscal ramp lesions: lessons learned from the past in the pursuit of evidence. Arthroscopy 34:1638-1640

41. Seil R, Mouton C, Coquay J, Hoffmann A, Nuhrenborger C et al (2018) Ramp lesions associated with ACL injuries are more likely to be present in contact injuries and complete ACL tears. Knee Surg Sports Traumatol Arthrosc 26:1080-1085

42. Slocum DB, Larson RL (1968) Rotatory instability of the knee. Its pathogenesis and a clinical test to demonstrate its presence. $\mathrm{J}$ Bone Jt Surg Am 50:211-225

43. Smith JP 3rd, Barrett GR (2001) Medial and lateral meniscal tear patterns in anterior cruciate ligament-deficient knees. A prospective analysis of 575 tears. Am J Sports Med 29:415-419

44. Song GY, Liu X, Zhang H, Wang Q-Q, Zhang J et al (2016) Increased medial meniscal slope is associated with greater risk of ramp lesion in noncontact anterior cruciate ligament injury. Am J Sports Med 44:2039-2046

45. Sonnery-Cottet B, Praz C, Rosenstiel N, Blakeney WG, Ouanezar $\mathrm{H}$ et al (2018) Epidemiological evaluation of meniscal ramp lesions in 3214 anterior cruciate ligament-injured knees from the SANTI study group database: a risk factor analysis and study of secondary meniscectomy rates following 769 ramp repairs. Am J Sports Med 46:3189-3197 
46. Stephen JM, Halewood C, Kittl C, Bollen SR, Williams A et al (2016) Posteromedial meniscocapsular lesions increase tibiofemoral joint laxity with anterior cruciate ligament deficiency, and their repair reduces laxity. Am J Sports Med 44:400-408

47. Strobel MJ (1988) Menisci. In: Fett HM, Flechtner P (eds) Manual of arthroscopic surgery. Springer, New York, pp 171-178

48. Tandogan RN, Taser O, Kayaalp A, Taskiran E, Pinar H et al (2004) Analysis of meniscal and chondral lesions accompanying anterior cruciate ligament tears: relationship with age, time from injury, and level of sport. Knee Surg Sports Traumatol Arthrosc 12:262-270

49. Tashiro Y, Mori T, Kawano T, Oniduka T, Arner JW et al (2020) Meniscal ramp lesions should be considered in anterior cruciate ligament-injured knees, especially with larger instability or longer delay before surgery. Knee Surg Sports Traumatol Arthrosc. https://doi.org/10.1007/s00167-020-06161-8

50. Thaunat M, Fayard JM, Guimaraes TM, Jan N, Murphy CG et al (2016) Classification and surgical repair of ramp lesions of the medial meniscus. Arthrosc Tech 5:e871-e875

51. Thaunat M, Jan N, Fayard JM, Kajetanek C, Murphy CG et al (2016) Repair of meniscal ramp lesions through a posteromedial portal during anterior cruciate ligament reconstruction: outcome study with a minimum 2-year follow-up. Arthroscopy 32:2269-2277

52. Warren LF, Marshall JL (1979) The supporting structures and layers on the medial side of the knee: an anatomical analysis. $J$ Bone Jt Surg Am 61:56-62

53. Wierer G, Milinkovic D, Robinson JR, Raschke MJ, Weiler A et al (2020) The superficial medial collateral ligament is the major restraint to anteromedial instability of the knee. Knee Surg Sports Traumatol Arthrosc. https://doi.org/10.1007/s00167-020-05947-0

54. Willinger L, Shinohara S, Athwal KK, Ball S, Williams A et al (2020) Length-change patterns of the medial collateral ligament and posterior oblique ligament in relation to their function and surgery. Knee Surg Sports Traumatol Arthrosc 28:3720-3732

55. Woods GW, Chapman DR (1984) Repairable posterior meniscocapsular disruption in anterior cruciate ligament injuries. Am J Sports Med 12:381-385

56. Yeo Y, Ahn JM, Kim H, Kang Y, Lee E et al (2018) MR evaluation of the meniscal ramp lesion in patients with anterior cruciate ligament tear. Skeletal Radiol 47:1683-1689

Publisher's Note Springer Nature remains neutral with regard to jurisdictional claims in published maps and institutional affiliations. 